\title{
DON JUAN UND KALTE LIEBE
}

\section{ABSTRACT. Don Juan and cold love}

The present article is an attempt to use the metaphorical category of temperature in the interpretation of literary creations of love in two different cultural contexts: against the background of the emotional culture of the inter-war period as well as of the present time. The analysis focusses on two literary texts: Don Juan kommt aus dem Krieg (1936) by Ödön von Horváth as well as Don Juan de la Mancha (2007) by Robert Menasse, which encourage to reflect on remarkable transformations of the culture of love and the positioning of both men and women within the new emotional styles.

KEYWORDS: Don Juan, gender, love, desire, temperature

\section{1.}

Heiße Liebe, entflammte Leidenschaften, glühende Erotik - in diesen Kategorien versucht man immer wieder dem Begehren und seinen Realisierungsformen Liebe und Sexualität beizukommen - sie zu imaginieren, zu beschreiben, zu inszenieren ${ }^{1}$. Erhitzungsszenarios als begleitende atmosphärische Kulisse von literarischen Verführungen haben eine lange Tradition, die die Vorstellungen von Glut und Feuer mit erotischen Ekstasen verknüpft und Männer gleichermaßen wie Frauen mit verschiedenen Begehrensmustern im Sinne von sozialen Codes, Regeln und Standards für das Erleben, die Intensitäten, nicht zuletzt die „Temperaturen“ der mikrosozialen emotionalen Arrangements konfrontiert. Schon in den antiken literarischen Texten wurde Kälte ,als Metapher genutzt, um den Verstand den 'heißen' Affekten und Begierden gegenüberzustellen“². Wärme und Kälte bezeichnen die Erscheinungsformen der Liebeskultur mit dem ihr zugrunde liegenden Gendering - einem

\footnotetext{
${ }^{1}$ Vgl. beispielsweise Octavio Paz: Die doppelte Flamme: Liebe und Erotik. Aus dem Spanischen von Rudolf Wittkopf. Frankfurt a. M. 1997.

${ }^{2}$ Ulla Haselstein: Coolness. In: Gunter Gebauer, Markus Edler (Hrsg.): Sprachen der Emotionen. Kultur, Kunst, Gesellschaft. Frankfurt a. M./New York 2014, S. 211-229, hier S. 217.
} 
Prinzip, nach dem kühle Selbstbeherrschung und habituelle Distanz bzw. ostentative Performanzen von Affektkontrolle grundsätzlich als „männlich“, und „heiße“ Leidenschaften bzw. heftige Aufwallungen von Gefühlen als „weiblich“ imaginiert und inszeniert werden. Doch diese „thermische“ Rollenbesetzung im Theater der Verführung, Erotik und Liebe ist nicht eine ontologische oder gar biologische Gegebenheit, sondern eben kulturell hervorgebracht und unterliegt folgerichtig - vor dem Hintergrund verschiedener Transformationen von Gefühlskulturen und Geschlechterverhältnissen - bedeutsamen historischen Wandlungen, die auch Männer der „Stimme ihres Herzens“ folgen und Frauen sich auf ihren kühlen Kopf in Liebesdingen berufen lassen. Diese Problematik kommt auf eine besonders signifikante Weise in der Figur des maßlosen Erotikers Don Juan zum Vorschein, der sowohl für heiße Leidenschaft als auch für flüchtige kalte Affären steht und durch die Leistungsdimension seiner ars amandi oder deren Gegenteil - die Abschwächung der erotischen Vitalität - in der jeweiligen Liebeskultur mehr oder weniger für Entrüstung, Irritation und Verwirrung sorgt. Dieser „spontan oder reflektiert verführende [...] Lust-

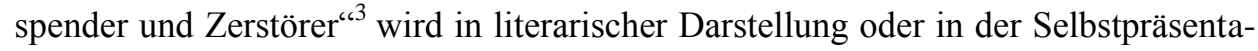
tion oft mithilfe einer Glut- und Feuermetaphorik charakterisiert und gleichzeitig von vielen Frauen als eiskalter Verführer, der sie ins Verderben stürzt, abgeurteilt. Auch die zahlreichen Interpretationen der Don-Juan-Texte aus verschiedenen Epochen betonen sowohl die Aspekte der ,heißen“ Verführung und erotischen Aktivität als auch männliche Kaltblütigkeit und Unfähigkeit zu lieben. In Bezug auf die älteren Text-Versionen bemerkt Denis de Rougemont schlicht: „Don Juans Taktik ist die Vergewaltigung, und sowie er erreicht hat, was er wollte, macht er sich auf und davon.“4 Der erotische Nomade sei nicht im Geringsten an einer Dauerhaftigkeit interessiert, sondern an kurzen Augenblicken des Genusses, und dies unterscheide ihn von Tristan, dem Rougemont „schlafwandlerische Langsamkeit" ${ }^{5}$ attestiert. Manfred Schneider betont das Prinzip Geschwindigkeit, das generell der „Arbeitsweise" des Verführers zugrunde liegt und Wärme eliminiert:

Eine Verführung, die Zeit in Anspruch nimmt, verfällt bald den Verzögerungen der Liebe, die ihre Zeit verzehrt und stillsteht. Ein Verführer, der donjuaneske Serien und Mengen von Eroberungen aufzählen will, ein Schauspieler oder ein Soldat der Liebe, dringt stets auf Tempo. Er erklärt alles zur Sache der Leidenschaft und stößt auf der anderen Seite nicht selten auf die Bereitschaft,

\footnotetext{
${ }^{3}$ Günter Helmes: „,Was geht mit mir vor? Wo bin ich?“ Eine Typologie deutschsprachiger DonJuan-Texte zwischen Lenau und Frisch. In: Helmut Kreuzer (Hrsg.): Don Juan und Femme fatale. München 1994, S. 59-97, hier S. 62.

${ }^{4}$ Denis de Rougemont: Don Juan und Tristan. Alternative oder Wechselbeziehung? In: Don Juan. Ein Lesebuch. Mit Texten von Max Frisch, E.T.A. Hoffmann, José y Ortega Gasset, Tirso de Molina, Albert Camus und vielen anderen Autoren. Hrsg. von Kurt-Jürgen Heering. München 1990, S. 46-52, hier S. 46.

${ }^{5}$ Ebd., S. 46.
} 
in der Showdown des sogenannten 'Spontanficks', den Erica Jong in die Literatur eingeführt hat, einzutreten. ${ }^{6}$

Sowohl in Don-Juan-Texten als auch in ihren Interpretationen gehen Liebesrhetorik und ,warme“" Gefühlswerte mit Begriffen aus den „kalten“ Bereichen der Kalkulation, Statistik ${ }^{7}$, Mathematik, Geometrie einher, wobei die letzteren zu dominieren scheinen. Jürgen Wertheimer beispielsweise stellt die „,buchhalterische Nüchternheit“ des auf „Massenkonsumprogramm“ ausgerichteten erotischen Serientäters heraus und konstatiert, dass Don Juan weit mehr „Fetischist der Statistik“ als Frauenliebhaber sei, der „perfiderweise“ nicht den Geschlechtsverkehr selbst genießt, sondern „dessen numerisch statistische Erfassung, seine Aufarbeitung, seine Dokumentation in der Endlosstrichliste, die Verwaltung von Sex in Text und Statistik ${ }^{\text {c8 }}$.

Es scheint interessant zu sein, Don Juans literarische Attitüden innerhalb der Wärme- und Kälte-Metaphorik zu diskutieren, allerdings unter Berücksichtigung der kulturellen Hintergründe, die jeweils durch eine charakteristische Gefühls- bzw. Liebeskultur geprägt sind. Das Ziel der vorliegenden Analyse ist es, literarische Inszenierungen der Liebe, die Julia Kristeva als „Gemisch aus zerstörerischem Besitz und Idealisierung “9 ${ }^{\text {(9) }}$ ezeichnet, im Kontext der Gefühlskulturkultur der Zwischenkriegszeit und der Gegenwart am Beispiel von zwei Aufarbeitungen des Don Juan-Stoffes, nämlich Ödön von Horváths Don Juan kommt aus dem Krieg (1936) und Robert Menasses Don Juan de la Mancha (2007) zu verfolgen. Die beiden Texte bieten einen Rückblick (bis zur Zeit unmittelbar nach dem Ersten Weltkrieg und bis in die 1960er Jahre) und provozieren somit eine Reflexion über die gravierenden Wandlungen der Liebeskultur oder - um es in meteorologischen Kategorien auszudrücken - über den emotionalen Klimawandel und die Positionierungen des Don Juan (und der Frauen) innerhalb der neuen affektiven Stile bzw. im Wind und Wetter der Liebe.

\section{2.}

Ödön von Horváth schildert einen Don Juan, der aus dem Großen Krieg kommt und in der Epoche der „Verhaltenslehren der Kälte“ - das heißt der Entemotionali-

\footnotetext{
${ }^{6}$ Manfred Schneider: Liebe und Betrug. Die Sprachen des Verlangens. München/Wien 1992, S. 193.

${ }^{7}$ Vgl. Mozarts Don Giovanni und seine Erfolge beim weiblichen Geschlecht, die in der berühmten Katalog-Arie aufgezählt werden (mille tre).

${ }^{8}$ Jürgen Wertheimer: Don Juan und Blaubart. Erotische Serientäter in der Literatur. München 1999, S. 20. Diese Interpretation bezieht sich insbesondere auf den intellektgesteuerten Don Juan-Typus des 18. Jahrhunderts.

${ }^{9}$ Julia Kristeva: Geschichten von der Liebe. Aus dem Französischen von Dieter Hornig und Wolfram Bayer. Frankfurt a. M. 1989, S. 63.
} 
sierung der Männlichkeit, Aufhebung der introspektiven Gewissenskultur und Orientierung am Muster der „kalten persona“"10 $-\mathrm{Fuß} \mathrm{zu}$ fassen versucht. Im Vorwort des Dramas deklariert der Autor: „Ich habe es mir also erlaubt, einen Don Juan unserer Zeit zu schildern [... $]^{\text {“11 }}$ - einer Zeit der großen Inflation, in der ,sich alle Werte verschoben haben“ (11). Diese extreme Instabilität und Unsicherheit fällt dem heimkehrenden kriegsbeschädigten Verführer besonders im Bereich der Geschlechterverhältnisse auf: Frauen und Männer sind anders geworden, das männliche Recht auf körperliche und ökonomische Überlegenheit gehört nicht mehr zu Selbstverständlichkeiten, die Frauen kommen als begehrende Subjekte zum Vorschein. In den Vorarbeiten zu dem Drama findet sich die folgende Passage (die Worte von Don Juan):

Manchmal denk ich in einer anderen Welt zu leben. Es hat sich alles geändert - - wenn ich mir überleg, wie vor dem Krieg die jungen Mädchen reagiert haben und jetzt? Ja, es ist auch ein Unterschied, ihr seid alle männlicher - - schon von der Statur aus. (96)

Die Frauen sind aktiv, emanzipiert, politisch engagiert, sie verlangen nach Mitbestimmungsrecht und lehnen die „Vorherrschaft des Mannes“ ab (21). Diese Transformationen bekommt der schwache, matte, „herzkranke“ ehemalige Soldat, der von einer Hölle zurückgekehrt ist ${ }^{12}$ und behauptet, ein anderer geworden zu sein, von einer Frau unmissverständlich erklärt: „Es wundert Sie, daß Sie eine Frau zum Tanzen auffordert, aber die Welt hat sich gedreht, mein Herr, und warum sollen nur die Männer Don Juane sein dürfen?“ (32) Einen weiteren Interpretationshinweis liefert wieder das Vorwort, in dem die Frage beantwortet wird, warum das Stück keine einzige Liebesszene enthält, nämlich deshalb, weil alle Frauen Don Juan erliegen, „aber - - und dies dürfte das Entscheidende sein: wirklich geliebt wird er von keiner“ (12). So wird der legendäre "heiße“ Verführer in der Nachkriegszeit mit „kalter“ liebloser Weiblichkeit konfrontiert, mit Frauen, die an männlicher Sexualität interessiert sind bzw. sich deren metaphysischer Wirkung nicht entziehen können (12).

Horváths Don Juan macht einen unsicheren Eindruck, fasst sich oft ans Herz, fühlt sich müde und sucht seine Verlobte, die er vor dem Krieg verlassen und der er viele Feldbriefe geschrieben hat. Er behauptet, dieser „Frau von gestern“, die eine vergangene Liebeskultur mit dem „warmen“ Medium des Liebesbriefs repräsentiert, seit dem Krieg treu zu sein: ,und diese Dame suche ich“ (18). Eine der Frauen erinnert an seine Vergangenheit einer ,stadtbekannte[n] Persönlichkeit mit lauter eroti-

\footnotetext{
${ }^{10}$ Vgl. Helmut Lethen: Verhaltenslehren der Kälte. Lebensversuche zwischen den Kriegen. Frankfurt a. M. 1994.

${ }^{11}$ Ödön von Horváth: Don Juan kommt aus dem Krieg. Frankfurt a. M. 2001, S. 11. Nach dieser Ausgabe werden die Zitate im Folgenden direkt im Text nachgewiesen.

${ }^{12}$ Vgl. die Interpretation von Ulrike Vedder: Krieg - Ehe - Krieg. Don Juan und die Kunst der Verführung im 20. Jahrhundert (Horváth, Frisch, Müller). In: Bogdan Mirtschev, Maja RazbojnikovaFrateva, Hans-Gerd Winter (Hrsg.): Mythos und Krise in der deutschsprachigen Literatur des 19. und 20. Jahrhunderts. Dresden 2004, S. 117-127.
} 
schen Skandalaffären“: Er habe seine Braut knapp vor der Hochzeit verlassen und „sich mit tausend miserablen Frauenzimmern herumgetrieben in Saus und Braus, derweil war seine Braut eine reine Seele, ein direkter Engel“" (19). Er kann die Braut nicht finden, weil sie aus Liebeskummer irrsinnig geworden und in einer Irrenanstalt gestorben ist, doch alle anderen erinnern ihn an diese Einzige, gewissermaßen an das verlorene Ideal, die große Liebe ${ }^{13}$, die er sich jetzt - er kann ja „den Damen nicht entrinnen“ (11) und beginnt neue Liebschaften - in fragmentierter Wahrnehmung „stückerlweise“ zusammensucht (43) ${ }^{14}$. Doch zeichnet sich dieser Don Juan, der als ein Sucher nach dem Ideal in romantischer Tradition bezeichnet werden könnte ${ }^{15}$, durch eine geradezu „unmännliche“ Passivität aus - im Sinne der tradierten kulturellen Imperative der Verführung, die verlangen, dass „der Mann die Eingangs-Worte spricht, um die Szene zu eröffnen und die Klimax der Blicke, Worte, Berührungen, Küsse bis hin zur Fleischwerdung anzustoßen “16. Es sind die Frauen, die die Initiative ergreifen, ihn verfolgen und erlösen wollen, denen er - der ,müde Kavalier“17 aber unheimlich erscheint (,Bei dir ist alles tot, als wär Glas zwischen uns [...].“ (57)). Der heimkehrende Soldat ist eben kein „Soldat der Liebe“, der stets aufs Tempo dringt, „die unmißverständliche Sprache der Macht“ spricht und den Imperativ bevorzugt - einen Modus, „der keine Stockung kennt ${ }^{\star 18}$ und allein auf Wirkungen hinausgeht. Er ist kein Held der „erotischen Abkürzungen und Beschleunigungen“, der sich „militärischer" Sprechakte bedient und lediglich Befehle und Drohungen ausspricht. Wenn die letzteren „Wirkungen erzielen, dann sind es Zustände zwischen Leben und Tod: Ergebung, Überwältigung, Ohnmacht, Bewußtlosigkeit“"19. Doch in Horváths Drama sind diese liminalen Zustände in das Schicksal des schwachen Verführers, der in Ohnmacht fällt und ins Krankenhaus eingeliefert wird, integriert.

In der letzten Szene des Dramas befindet sich der „Schuft“, der „wiedergutmachen" wollte (69), in einer nächtlichen Schneelandschaft auf dem Friedhof und spricht zu seiner toten Braut: „Ist es dir kalt, wenn es schneit? Soll ich zu dir kommen? - - - Ja, ich werde dich immer suchen, als würdest du leben.“ (71) Es schneit ununterbrochen und Don Juan wird ,der reinste Schneemann“ (71), der leise lächelt, sich einschneien lässt und Abschied nimmt mit den Worten: „es wird immer wärmer“ (72). Schnee und Kälte sorgen für atmosphärische Verdichtung, für „Präsenz-

\footnotetext{
${ }^{13}$ In den Vorarbeiten zum Drama heißt es: „,meine große seelische Liebe“ (115).

${ }^{14}$ Am Ende wird er eines Missbrauchs einer Minderjährigen beschuldigt und muss von der Stadt fliehen.

${ }^{15}$ Vgl. Hiltrud Gnüg: Don Juan. Ein Mythos der Neuzeit. Bielefeld 1993, S. 95.

${ }^{16}$ Schneider: Liebe und Betrug, S. 14.

${ }^{17}$ Johanna Bossinade: Vom Kleinbürger zum Menschen. Die späten Dramen von Ödön von Horváth. Bonn 1988, S. 11.

${ }^{18}$ Schneider: Liebe und Betrug, S. 28.

${ }^{19}$ Ebd., S. 238.
} 
effekte $^{\text {‘20 }}$, die in ihrer Vergänglichkeit von Absenz durchdrungen sind, und bilden einen Ausgangspunkt für verschiedene Interpretationen („,Bedeutungseffekte“), die sich unter anderem auf die Intensitäten des Begehrens, die erotische Heimatlosigkeit, die Nähe des Todes oder die Suche nach Geborgenheit beziehen. Der Schnee kann nicht nur mit einer kalten Dimension assoziiert, sondern auch als wärmende Schutzhülle imaginiert werden, was für manche Interpreten das Phantasma des schützenden Mutterleibes symbolisiert ${ }^{21}$. Gleichwohl wird Wärme vom „müden Schneemann“ Don Juan, der auf dem Friedhof auf einem Stein sitz ${ }^{22}$, als eine Erlösungsperspektive (Vereinigung mit der Geliebten?) evoziert. Die Ambivalenz der klimatisch-jahreszeitlichen Staffage provoziert viele Fragen, denn der Schnee ist „,nicht mit sich selbst identisch“ und kann „Wärme gleichermaßen wie Kälte, Leben wie Tod, Freude wie Trauer, Kindheit wie Alter “23 symbolisieren. Auch Don Juan ist nicht mehr mit sich selbst identisch - weder mit seiner Vorkriegs-Attitüde noch mit der Legende der literarischen Tradition. Er verschwindet unter Schnee und gewissermaßen von der Bühne, auf der er sich bis dahin mehr oder weniger in der Rolle eines „heißen“"Verführers und „kalten“ Liebhabers bewährte.

\section{3.}

Diesen Abschied markiert noch gravierender die Komödie Don Juan oder die Liebe zur Geometrie (1953) von Max Frisch, in der der legendäre Verführer dem eigenen Mythos zu entkommen sucht und nicht mehr Frauen, sondern die Geometrie liebt, denn letztere sei - im Gegensatz zum „Sumpf unserer Stimmungen“ - ein „Wissen, das stimmt “24, und von keinen Launen abhängt. Vielleicht ist es kein Zufall, dass er sich ausgerechnet für das Dreieck als eine in seiner Eindeutigkeit geradezu „feierliche“ Figur begeistert:

So und nicht anders! Sagt die Geometrie. So und nicht irgendwie! Da hilft kein Schwindel und keine Stimmung, es gibt eine einzige Figur, die sich mit ihrem Namen deckt. Ist das nicht schön? (132)

In Nachträgliches zu „Don Juan “ charakterisiert Max Frisch seinen (Anti-)Helden als einen lieblosen Intellektuellen mit dem Anspruch auf eine männliche Geistigkeit und einer Angst vor dem Weiblichen in sich selbst:

\footnotetext{
${ }^{20}$ Hans Ulrich Gumbrecht: Präsenz. Hrsg. von Jürgen Klein. Berlin 2012, S. 340.

${ }^{21}$ Vgl. beispielsweise Ingrid Haag: Der weiße Mantel der Unschuld. Dramatische Textur des Horváthschen Motivs. In: Klaus Kastberger (Hrsg.): Unendliche Dummheit - dumme Unendlichkeit. Wien 2001, S. 63-73, hier S. 65.

${ }^{22}$ Dieses Bild erinnert natürlich an den steinernen Gast.

${ }^{23}$ Andrea Dortmann: Winter Facets. Traces and Tropes of the Cold. Bern 2007, S. 10.

${ }^{24}$ Max Frisch: Don Juan oder die Liebe zur Geometrie. Komödie in fünf Akten. In: ders.: Gesammelte Werke in zeitlicher Folge (1931-1985). Bd. 3. Hrsg. von Hans Mayer unter Mitwirkung von Walter Schmitz. Frankfurt a. M. 1986, S. 96-175, hier S. 131.
} 
Liebe, wie Don Juan sie erlebt, muß das Unheimlich-Widerliche der Tropen haben, etwas wie feuchte Sonne über einem Sumpf voll blühender Verwesung, panisch, wie die klebrige Stille voll mörderischer Überfruchtung, die sich selbst auffrißt, voll Schlinggewächs - ein Dickicht, wo man ohne blanke Klinge nicht vorwärtskommt; wo man Angst hat zu verweilen. ${ }^{25}$

Wärme und Feuchtigkeit stehen hier für die Unerträglichkeit der Liebesnähe und die Unvorhersehbarkeit der mit hohen Temperaturen assoziierten emotionalen Arrangements, die - im Gegensatz zur objektiven und verständlichen Geometrie - sich der Kontrolle entziehen und Orientierungslosigkeit stiften. In Barbara Honigmanns Erzählung Don Juan (1984) wird eine bemerkenswerte Regie geführt, die die Oppositionen von Frisch umkehrt: Hier will der „höhnische Verbrecher“, nach vielen erlebten Abenteuern seines Nomadentums überdrüssig geworden, von Donna Anna in Sevilla aufgenommen und endlich sesshaft werden, sie jedoch - sehnt sich nach dem Fremden und nach weiten Reisen. Der Konflikt endet mit dem Mord an der Geliebten und Don Juan wird auf dem Friedhof dargestellt, wo er mit einer Frauenstatue spricht: „Ich möchte dich heiraten. Ich möchte dein Mann sein. Mit dir will ich Hochzeit feiern. Willst du mich nicht?“ ${ }^{26}$ Er schmückt sie mit Laub und Zweigen, legt ihr ein Brautkleid an, lädt sie zum Hochzeitsmahl ein, bittet den Himmel um Blitz, Donner und Regen, damit das Fest mit Licht, Musik und Wein gefeiert wird, und wendet sich an die Geliebte: „Komm, meine Braut. Jetzt will ich dich umarmen. Wir wollen sehen, ob du mich kalt machst oder ich dich erwärmen kann. “27

Der Protagonist des Romans Don Juan de la Mancha von Robert Menasse wird mit anderen Problemen konfrontiert, doch wenn man die Geschichte des literarischen Mythos Don Juan verfolgt, lässt sich feststellen, dass sich der Übergang vollzogen hat „von einem Begehren, das sich aus dem Mangel speist, zu einem Begehren, das der Überfülle des Seins geschuldet ist ${ }^{\text {‘2 }}$. Der über 50-jährige Nathan führt ein exzessives Sexualleben, fühlt sich aber extrem gelangweilt. Er verspürt keine Lust - und dies in der Zeit der sexuellen Befreiung und des tabulosen Umgangs mit Erotik und Sexualität:

Es gibt wahrscheinlich keinen Antrieb, der so gewaltig ist wie der, der einem Mann zu glühen beginnt, wenn er die Lust verloren hat in einer Gesellschaft, die nicht einmal einen Liter Mineralwasser verkaufen kann, ohne diese Ware erotisch zu besetzen. ${ }^{29}$

Das Lustprinzip ist allgegenwärtig, das „Sein ist Erregtsein“ ${ }^{\text {(30 }}$ geworden, trotzdem oder gerade deswegen braucht der vermeintliche Lustheros eine Therapeutin, die

\footnotetext{
${ }^{25}$ Ebd., S. 169.

${ }^{26}$ Barbara Honigmann: Don Juan. In: Don Juan. Geschichten zwischen Liebe und Tod. Hrsg. von Birgit Heiderich und Frank Göhre. Reinbek bei Hamburg 1986, S. 45-52.

${ }^{27}$ Ebd., S. 52.

${ }^{28}$ Angerer: Vom Begehren nach dem Affekt, S. 8.

${ }^{29}$ Robert Menasse: Don Juan de la Mancha oder Die Erziehung der Lust. Roman. Frankfurt a. M. 2009, S. 8. Nach dieser Ausgabe werden die Zitate im Folgenden direkt im Text nachgewiesen.
} 
sich der Herausforderung stellt, seine Lust zu rekonstruieren: „Was Sie an Ihrer Frau haben, wissen Sie. Aber was Sie nicht haben, können Sie nur bei anderen suchen. Das ist eine Frage der Logik und nicht der Moral!“ (15) Die Sphäre der Intimität wurde gründlich entzaubert und damit zu einer Domäne der Rationalität - eine Tendenz, die von Eva Illouz systematisch untersucht wird ${ }^{31}$. Die Ursachen dieser Entzauberung der Liebe finden allesamt Eingang in Menasses Roman: Dominanz der wissenschaftlichen Erklärungsversuche, rationalisierte und marktförmig organisierte Partnersuche im Internet und der Feminismus, der einen ,wichtigen Einschnitt in die Geschichte der Liebe“ markiert, „weil er den Schleier des männlichen Rittertums und den geheimnisvollen weiblichen Nimbus herunterriss, der Frauen gleichzeitig zu Sklaven und Gottheiten und Männer zu Peinigern und Rettern machte “'32. Nathan hat auch die Liebe übers Internet getestet und 436 Fragen zu seiner Persönlichkeit beantwortet, die eine introspektive Selbstprüfung voraussetzen und eine auf rationalem Vergleich und auf Abwägen von Optionen basierende Entscheidung ermöglichen sollten ${ }^{33}$. Es gelingt ihm nicht, ,das höchste Ziel: Lust“ (39) zu erreichen, obwohl er es mit verschiedenen Frauen versucht hat und immer noch versucht:

eines Tages, wenn meine Körperpanzer brachen, würde ich eine Lust empfinden, so groß, dass es mich gleichsam ins All zerstäubte. Technisch war schon alles perfekt. Es musste nur noch - was? (45)

Verschiedene Erklärungsversuche werden ins Feld geführt: Christa meint, ihm würde Befriedigung nicht genügen, sondern er brauche Erlösung (104), ein Arbeitskollege spricht von seinem Zynismus und dem Kalauern, das „immer eine AntiStimmung“ erzeuge - eine ,atmosphärische Gegnerschaft zu allem“ (119). Und Nathan selbst, der permanent über das Rätsel Lust räsoniert, kommt zu einer Diagnose über die Generation, die gesellschaftliche Zwänge und Konventionen in der Intimsphäre abzuschütteln suchte, sich aber gleichzeitig „so angestrengt um Kälte und analytische Schärfe“ bemühte (147). Auch nach der sexuellen Revolution hatte sich die Liebe anderen Prinzipien zu fügen (,wir haben uns nur als Dienstleister neu definiert“ (138)): „unsere Liebe musste auch dienen - der Frauenbefreiung, der

${ }^{30}$ Norbert Bolz: Das Begehren und der Konsum. In: Peter Kemper, Ulrich Sonnenschein (Hrsg.): Das Abenteuer Liebe. Bestandsaufnahme eines unordentlichen Gefühls. Frankfurt a. M. 2004, S. 233-239, hier S. 234.

${ }^{31}$ Vgl. beispielsweise ihre Arbeiten: Der Konsum der Romantik. Liebe und die kulturellen Widersprüche des Kapitalismus. Aus dem Amerikanischen von Andreas Wirthensohn. Frankfurt a. M. 2007 und: Warum Liebe weh tut. Eine soziologische Erklärung. Aus dem Englischen von Michael Adrian. Berlin 2012.

${ }^{32}$ Eva Illouz: Eine Religion ohne Glauben: Liebe und die Ambivalenz der Moderne. Aus dem Englischen von Simone Scheps. In: Yvonne Niekrenz, Dirk Villányi (Hrsg.): LiebesErklärungen. Intimbeziehungen aus soziologischer Perspektive. Wiesbaden 2008, S. 211-220, hier S. 217.

${ }^{33}$ Es geht letztlich darum, ein potentielles Objekt in seine Bestandteile zu zerlegen, was die emotionale oder intuitive Kraft von Entscheidungen deutlich verkümmern lässt. 
Emanzipation von Geschlechterrollen, und letztlich auch Gott“"(138) als einem Ideal der Liebe im Sinne der Prinzipien von Verzeihung und Strafe.

In Nathans Selbstbespiegelungsnarration ist immer wieder die Rede von Liebe, auch zu seiner zweiten Ehefrau, der er eine SMS mit der abgenutzten Phrase „Ich liebe dich“ schickt und natürlich die Antwort „Ich dich auch“ bekommt. Doch Liebe „führt konsequent zu getrennten Schlafzimmern“ (186), deshalb sucht Nathan Glück in kurzlebigen Affären ${ }^{34}$, obwohl er ja auch eine Langzeitgeliebte hat, mit der er immerhin - darin wirklich Don Juan - „den kleinen Tod“ erleben kann (104). Sein Problem versucht er folgendermaßen zu definieren:

Eine Frau war für ihn leicht 'zu haben', aber eben nicht das Glück. Wenn er eine Frau kennenlernte, war er gespannt wie vor einer Theaterpremiere. Er liebte das Theater. Dann ging der Vorhang auf. $\mathrm{Zu}$ viel Outrieren. $\mathrm{Zu}$ viel Bühnenbild. $\mathrm{Zu}$ viel Eitelkeit. $\mathrm{Zu}$ viel Nachtarbeit. So war das Theater. Seine Affären. (243)

In Paris stellt er fest, dass alte Liebesklischees nicht viel taugen und dass ihn „kühl blickende“ Französinnen mehr anziehen als „die offen ausgestellte Sinnlichkeit der Brasilianerinnen“ (169). „L'amour hat eben noch einen anderen Klang als das ausgelutschte amor, amore oder gar love.“ (169)

Liebe, Begehren und Sexualität erscheinen als komplett getrennte Kategorien, zu denen Nathan - nicht zuletzt durch Ironie, Sarkasmus und einen Umschlag in die Erzählung in dritter Person - Distanz zu gewinnen sucht. In seinem Lebenslauf spiegeln sich diverse Muster der postmodernen Gefühlskultur: sowohl die kleinen Liebesnarrationen mit ihren „lokal begrenzten und flüchtigen Intensitäten“ als auch langfristige „globale“ Liebeserzählungen - wie etwa die Ehe als ein übergreifendes Narrativ dauerhafter Liebe ${ }^{35}$. Das ironische Selbstmisstrauen geht mit dem therapeutischen Bedürfnis nach Wiederherstellung der Lust ${ }^{36}$ einher und manifestiert sich in der Sprache der Liebe, der ein Bewusstsein des Zitathaften und Sekundären jedes erotischen Arrangements als standarisierter Reproduktion gesellschaftlichen Codes inhärent ist, wie etwa in folgender Passage:

${ }^{34}$ Seine „Sammlerleidenschaft“ wird auch als eine „,kompensatorische Erfindung“ interpretiert, und zwar ,angesichts seines Misserfolges als entlassener Journalist, während seine Frau, mit der er keinen Sex hat, beruflich die Karriereleiter emporklettert“ (Brigitte Schwens-Harrant: Chili-Schoten und Geschlechter-Kämpfe: Beziehungskisten bei Robert Menasse und Michael Stavaric. In: Stefan Neuhaus (Hrsg.): Figurationen der Liebe in Geschichte und Gegenwart, Kultur und Gesellschaft. Würzburg 2012, S. 293-306, hier 299).

${ }^{35}$ Eva Illouz: Zur postmoderner Lage der Liebe. In: Peter Kemper, Ulrich Sonnenschein (Hrsg.): Das Abenteuer Liebe. Bestandsaufnahme eines unordentlichen Gefühls. Frankfurt a. M. 2004, S. 251-259, hier S. 257.

${ }^{36}$ Norbert Bolz macht auf das Angebot der Ratgeberliteratur aufmerksam, das mit den zahlreichen „scripts of desire“ vorgibt, alle Probleme der Intimität lösen zu können: „Sex wird langweilig, wenn es nicht gelingt, die sexuelle Variabilität und die Komplexität der Liebesbeziehung durch ein erotisches Training zu steigern.“ (Norbert Bolz: Das konsumistische Manifest. München 2002, S. 134). 
Endlich begriff ich, dass ich Beate begehrte - was ich begriff, war, dass ich sie begehrte, obwohl ich nicht beim ersten Blick ans Bett gedacht hatte. Klingt das bieder, romantisch oder gar kitschig? (257)

Und noch ein Beispiel:

Bitte, Hannah! Wie soll ich denn sagen? Liebe machen ist spießig und außerdem völlig verlogen, weil ich ja nicht Liebe meine, sondern Lust. Geschlechtsverkehr ausüben? Zeugungsadjustierung exekutieren?/ Ficken./ Okay. (139)

Was Hiltrud Gnüg über die Inszenierungen der „Eroberungen“ des extensiven Verführers Don Juan in der literarischen Tradition konstatiert, gilt auch für Menasses Roman, obwohl Nathan nicht unbedingt auf rasche Befriedigung seines Begehrens zielt, sondern sich auch wirklich Zeit nimmt: Don Juan neigt ,kaum zu schwelgerischen erotischen Beschreibungen weiblicher Reize, wollüstiger Gefühle, der Empfindungsskalen erotischer Lust ${ }^{\star 37}$. Schon wieder bringt der Don Juan-Mythos eine Literatur hervor, die ,die spontane, unreflektierte Sinnlichkeit gerade bezweifelt، ${ }^{\text {38 }}$, obwohl nun keine Hindernisse vorzuliegen scheinen:

Alles, was früher die Liebenden beklagten, die wachsamen Eltern, die Konventionen der Geschlechterbeziehung, der Zwang zur Ehe, sind abgeschafft, Freiheit, Gleichberechtigung, Kontrazeptiva, Telefon, Mail haben Sex und Liebe souverän werden lassen. Und doch scheint das Spiel schwieriger geworden zu sein. ${ }^{39}$

Und Menasses Don Juan, der kulturspezifische Spielarten der Liebe im Zeitalter der Coolness ${ }^{40}$ vorführt, in dem man nichts direkt sagen kann, in der Epoche der „,coolen Poesie“, „die mit allen Mitteln vermeidet, das Sexuelle auszusprechen“41 , stellt die grundsätzliche Frage: „Warum kann man nicht genießen?“ (16) Seit vierzehn Jahren verheiratet, mit steiler Karriere seiner Ehefrau konfrontiert, „Spezialist für frustrierte verheiratete Frauen“ (263) geworden, seit zwei Jahren in der Therapie, vor einigen Tagen entlassen, gelingt es ihm, in einem Reformhaus Glück zu entdecken:

Es heißt 'Bellamnion' und sieht aus wie rötlichbraunes Salz. Zwei Löffel davon in die Badewanne bewirken, dass das Badewasser dieselbe chemische Zusammensetzung wie Fruchtwasser hat. Es enthält Elektrolyte, Glucose, Lipide, Proteine und Eiweiß in genau der Menge und dem Verhältnis, wie die Flüssigkeit, in der wir neun Monate lang geschwommen sind. (203-204)

${ }^{37}$ Gnüg: Don Juan, S. 191.

${ }^{38}$ Ebd., S. 193.

${ }^{39}$ Manfred Schneider: Herzensschriften. Liebesbriefe und Liebesroman: In: Peter Kemper, Ulrich Sonnenschein (Hrsg.): Liebe zwischen Sehnsucht und Simulation. Frankfurt a. M. 2005, S. 276-286, hier S. 284.

${ }^{40}$ Zur kulturellen Karriere der Coolness vgl. Haselstein: Coolness.

${ }^{41}$ Schneider: Herzensschriften, S. 286. 
Die Pharmaindustrie verbucht hier einen literarischen Erfolg, der darauf hinausläuft, eine emotionale Tönung des Raums (eine Stimmung) oder mehr - einen affektiven Zustand zu erzeugen, der - zusammen mit der entsprechenden Temperatur des Wassers - für das Glücksgefühl sorgt, was der „erkaltete“ Eros ${ }^{42}$ nicht mehr leistet: „Ich überprüfte die Wassertemperatur mit dem Badethermometer, ließ heißes Wasser nachrinnen, berechnete den Grad der Verdünnung und ergänzte die entsprechende Menge Ballamnion [...]“ (204). In dieser Atmosphäre erinnert sich der 50-jährige Mann im Fruchtwasser an seine Frauen und räsoniert über vergangene Lusterlebnisse, wohl im Bewusstsein, dass es sich nicht zuletzt um seine Männerphantasien handelt: „Ich lag in der Badewanne und war glücklich.“(204)

\section{4.}

Horváths und Menasses Don Juan-Figuren sind literarische Produkte von Gefühlskulturen, für die eine jeweils spezifische Affirmation der Kälte charakteristisch ist, mit der „die verborgene Passion ${ }^{\text {“43 }}$ der Aufwertung von Geld, Karriere, Status sowie ein Mangel an festen Codierungen von Intimität und eindeutigen Geschlechtermustern einhergehen. Die dadurch herbeigeführte Orientierungslosigkeit in der Epoche der „sachlichen Romanzen“ (E. Kästner) ${ }^{44}$ und in der Zeit der „reinen Beziehungen“ (A. Giddens) ${ }^{45}$ führt zu rückwärtsgewandten Sehnsüchten und Reflexionen sowie zu Diagnosen über die gegenwärtige Lage, in der sich das weibliche Begehren das Existenzrecht verschafft und die Lust als männliche Macht die Vorrangstellung einbüßt. Der (modernen) leicht melancholischen Sehnsucht nach der verlorenen Geliebten, dem vergeblichen Suchen und Warten, der Evokation von abwesender großer Liebe und dem Tod im Schnee als Erlösung stehen die (postmodernen) Defizite und Illusionen gegenüber: Mangel an Begehren, Langeweile beim Sex, Suche nach Erfüllung im „kleinen Tod“, künstliche Erzeugung von Glück im Rahmen der Selbsthilfekultur. Don Juan - ein Sinnbild freien erotischen Genusses und Urbild des Verführers - braucht nicht mehr gegen Tabus und Verbote zu rebellieren, sondern ist mit Lustlosigkeit und Langeweile konfrontiert und sucht sich durch seine, durchaus zeittypische, kühle Intimitätsökonomie - vor „Unlust in Form

\footnotetext{
${ }^{42}$ Vgl. Rüdiger Safranski: Der erkaltete Eros. In: Moritz Baßler, Ewout van der Knaap (Hrsg.): Die (k)alte Sachlichkeit. Herkunft und Wirkungen eines Konzepts. Würzburg 2004, S. 9-17.

${ }^{43}$ Peter von Matt: Liebesverrat. Die Treulosen in der Literatur. München/Wien 1991, S. 381. Von Matt spricht in diesem Zusammenhang von der Verlogenheit der „Phrase der Liebe“ und der historischen Transformation der Liebe, die „Bestandteil der Selbstwerdung im sozialen Aufstieg“ wird.

${ }^{44}$ Vgl. Erich Kästner: Sachliche Romanze. In: Das große Erich Kästner Lesebuch. Hrsg. von Sylvia List. München 2008, S. 115.

${ }^{45}$ Anthony Giddens: Wandel der Intimität: Sexualität, Liebe und Erotik in modernen Gesellschaften. Aus dem Englischen Hanna Pelzer. Frankfurt a. M. 1993, S. 69.
} 
von Enttäuschung und Schmerz“46 zu schützen. Sexualität spielt in seiner biographischen Narration eine entscheidende Rolle, ist sie doch eine der wichtigsten Arenen geworden, in der Männlichkeitsstatus demonstriert wird. Gepaart mit Serialität ,verhilft sie zu einem größeren strategischen Vorteil und zu mehr Macht im Bereich der Gefühle als die Ausschließlichkeitsstrategie ${ }^{\text {“47 }}$, obwohl das Prinzip der Serie andererseits mit emotionaler Distanziertheit einhergeht.

Die klimatisch-atmosphärische und landschaftlich-topographische Kulisse, die wenn auch temporär und transitorisch - bei Horváth eine Rolle spielt (Kälte und Wärme, Schnee und Sonne), wird bei Menasse pharmakologisch bzw. technisch hergestellt und sorgt für eine Stimmung, die bezahlbar, wiederholbar und verlängerbar ist. Die Fragilität des durch den Krieg gebrochenen, müden und schwachen Don Juan ohne Eigenschaften, der unglücklich ist, weil er die Frau verloren hat, findet eine Entsprechung in der Lustlosigkeit und Angst vor Risiko, Schmerz, Enttäuschung des Don Juan de la Mancha, der unglücklich ist, weil er das Begehren verloren hat. Doch endet die erste Geschichte, wie die meisten Don Juan-Inszenierungen - mit einem Desaster, und die zweite mit weiteren Versuchen, die eigenen Gefühle cool zu managen, mehr oder weniger intimitätsfreie Nähe zu Frauen zu suchen, gefühlsneutralen und bindungslosen Sex mit ihnen zu haben, die Haltung der Distanz und Ironie als Gegenteil von Intensitäten zu bewahren, höchstens lauwarme Temperaturen des Begehrens zu erreichen. Nathan wird die „,erotische Sisyphusarbeit“48 sein Streben nach Liebesglück - wie einen Kampf gegen die Windmühlen fortsetzen, solange sein Begehren nach dem Begehren nicht erlöscht.

In diesem Sinne ist Don Juan ein Massenprodukt der gegenwärtigen Gefühlskultur, in der „sukzessive Polygamie“49 ein weitgehend akzeptiertes Beziehungsmuster darstellt. So nimmt es nicht Wunder, dass Nathans Therapeutin in Menasses Roman am Bestseller Das Don Juan-Syndrom arbeitet, um das Spektrum der Ratgeberliteratur bzw. das Leistungspotential der „Therapie-, Selbsthilfe- und Coachingkultur ${ }^{\text {‘50 }}$ zu bereichern und vielen ausgekühlten, abgeklärten, illusionslosen Männern (und Frauen) zur „Optimierung“ ihrer Beziehungen zu verhelfen.

\section{Literatur}

Bolz, Norbert: Das Begehren und der Konsum. In: Peter Kemper, Ulrich Sonnenschein (Hrsg.): Das Abenteuer Liebe. Bestandsaufnahme eines unordentlichen Gefühls. Frankfurt a. M. 2004, S. 233-239.

\footnotetext{
${ }^{46}$ Gerda E. Moser: Porn s-kills. Was Robert Menasses „Nathan” und ein „Sextourist” zu erzählen haben - ein gewagter Vergleich. In: Doris Moser, Kalina Kupczyńska (Hrsg.): Die Lust im Text. Eros in Sprache und Literatur. Wien 2009, S. 389-397, hier S. 396.

${ }^{47}$ Illouz: Warum Liebe weh tut, S. 195.

${ }^{48}$ Wertheimer: Don Juan und Blaubart, S. 58.

${ }^{49}$ Bolz: Das Begehren und der Konsum, S. 239.

${ }^{50}$ Illouz: Warum Liebe weh tut, S. 204.
} 
Bossinade, Johanna: Vom Kleinbürger zum Menschen. Die späten Dramen von Ödön von Horváth. Bonn 1988.

Dortmann, Andrea: Winter Facets. Traces and Tropes of the Cold. Bern 2007.

Frisch, Max: Don Juan oder die Liebe zur Geometrie. Komödie in fünf Akten. In: ders.: Gesammelte Werke in zeitlicher Folge (1931-1985). Bd. 3. Hrsg. von Hans Mayer unter Mitwirkung von Walter Schmitz. Frankfurt a. M. 1986, S. 96-175.

Giddens, Anthony: Wandel der Intimität: Sexualität, Liebe und Erotik in modernen Gesellschaften. Aus dem Englischen Hanna Pelzer. Frankfurt a. M. 1993.

Gnüg, Hiltrud: Don Juan. Ein Mythos der Neuzeit. Bielefeld 1993.

Gumbrecht, Hans Ulrich: Präsenz. Hrsg. von Jürgen Klein. Berlin 2012.

Haag, Ingrid: Der weiße Mantel der Unschuld. Dramatische Textur des Horváthschen Motivs. In: Klaus Kastberger (Hrsg.): Unendliche Dummheit - dumme Unendlichkeit. Wien 2001, S. 63-73.

Haselstein, Ulla: Coolness. In: Gunter Gebauer, Markus Edler (Hrsg.): Sprachen der Emotionen. Kultur, Kunst, Gesellschaft. Frankfurt a. M./New York 2014, S. 211-229.

Helmes, Günter: „,Was geht mit mir vor? Wo bin ich?" Eine Typologie deutschsprachiger Don-JuanTexte zwischen Lenau und Frisch. In: Helmut Kreuzer (Hrsg.): Don Juan und Femme fatale. München 1994, S. 59-97.

Honigmann, Barbara: Don Juan. In: Birgit Heiderich und Frank Göhre (Hrsg.): Don Juan. Geschichten zwischen Liebe und Tod. Reinbek bei Hamburg 1986, S. 45-52.

Horváth, Ödön von: Don Juan kommt aus dem Krieg. Frankfurt a. M. 2001.

Illouz, Eva: Der Konsum der Romantik. Liebe und die kulturellen Widersprüche des Kapitalismus. Aus dem Amerikanischen von Andreas Wirthensohn. Frankfurt a. M. 2007.

Illouz, Eva: Eine Religion ohne Glauben: Liebe und die Ambivalenz der Moderne. Aus dem Englischen von Simone Scheps. In: Yvonne Niekrenz, Dirk Villányi (Hrsg.): LiebesErklärungen. Intimbeziehungen aus soziologischer Perspektive. Wiesbaden 2008, S. 211-220.

Illouz, Eva: Warum Liebe weh tut. Eine soziologische Erklärung. Aus dem Englischen von Michael Adrian. Berlin 2012.

Illouz, Eva: Zur postmoderner Lage der Liebe. In: Peter Kemper, Ulrich Sonnenschein (Hrsg.): Das Abenteuer Liebe. Bestandsaufnahme eines unordentlichen Gefühls. Frankfurt a. M. 2004, S. 251-259.

Kästner, Erich: Sachliche Romanze. In: Das große Erich Kästner Lesebuch. Hrsg. von Sylvia List. München 2008.

Kristeva, Julia: Geschichten von der Liebe. Aus dem Französischen von Dieter Hornig und Wolfram Bayer. Frankfurt a .M. 1989.

Lethen, Helmut: Verhaltenslehren der Kälte. Lebensversuche zwischen den Kriegen. Frankfurt a. M. 1994.

Matt, Peter von: Liebesverrat. Die Treulosen in der Literatur. München/Wien 1991.

Menasse, Robert: Don Juan de la Mancha oder Die Erziehung der Lust. Roman. Frankfurt a. M. 2009.

Moser, Gerda E.: Porn s-kills. Was Robert Menasses „Nathan” und ein „Sextourist” zu erzählen haben - ein gewagter Vergleich. In: Doris Moser, Kalina Kupczyńska (Hrsg.): Die Lust im Text. Eros in Sprache und Literatur. Wien 2009, S. 389-397.

Paz, Octavio: Die doppelte Flamme: Liebe und Erotik. Aus dem Spanischen von Rudolf Wittkopf. Frankfurt a. M. 1997.

Rougemont, Denis de: Don Juan und Tristan. Alternative oder Wechselbeziehung? In: Don Juan. Ein Lesebuch. Mit Texten von Max Frisch, E.T.A. Hoffmann, José y Ortega Gasset, Tirso de Molina, Albert Camus und vielen anderen Autoren. Hrsg. von Kurt-Jürgen Heering. München 1990, S. 46-52.

Safranski, Rüdiger: Der erkaltete Eros. In: Moritz Baßler, Ewout van der Knaap (Hrsg.): Die (k)alte Sachlichkeit. Herkunft und Wirkungen eines Konzepts. Würzburg 2004, S. 9-17. 
Schneider, Manfred: Herzensschriften. Liebesbriefe und Liebesroman: In: Peter Kemper, Ulrich Sonnenschein (Hrsg.): Liebe zwischen Sehnsucht und Simulation. Frankfurt a. M. 2005, S. 276-286.

Schneider, Manfred: Liebe und Betrug. Die Sprachen des Verlangens. München/Wien 1992.

Schwens-Harrant, Brigitte: Chili-Schoten und Geschlechter-Kämpfe: Beziehungskisten bei Robert Menasse und Michael Stavaric. In: Stefan Neuhaus (Hrsg.): Figurationen der Liebe in Geschichte und Gegenwart, Kultur und Gesellschaft. Würzburg 2012, S. 293-306.

Vedder, Ulrike: Krieg - Ehe - Krieg. Don Juan und die Kunst der Verführung im 20. Jahrhundert (Horváth, Frisch, Müller). In: Bogdan Mirtschev, Maja Razbojnikova-Frateva, Hans-Gerd Winter (Hrsg.): Mythos und Krise in der deutschsprachigen Literatur des 19. und 20. Jahrhunderts. Dresden 2004, S. 117-127.

Wertheimer, Jürgen: Don Juan und Blaubart. Erotische Serientäter in der Literatur. München 1999. 